\title{
Inhibitory Activities of Stauntonia hexaphylla Leaf Constituents on Rat Lens Aldose Reductase and Formation of Advanced Glycation End Products and Antioxidant
}

\author{
Seung Hwan Hwang, ${ }^{1}$ Shin Hwa Kwon, ${ }^{2}$ Set Byeol Kim, ${ }^{1}$ and Soon Sung Lim ${ }^{1,2,3}$ \\ ${ }^{1}$ Department of Food Science and Nutrition, Hallym University, 1 Hallymdeahak-gil, Chuncheon 24252, Republic of Korea \\ ${ }^{2}$ Institute of Natural Medicine, Hallym University, 1 Hallymdeahak-gil, Chuncheon 24252, Republic of Korea \\ ${ }^{3}$ Institute of Korean Nutrition, Hallym University, 1 Hallymdeahak-gil, Chuncheon 24252, Republic of Korea \\ Correspondence should be addressed to Soon Sung Lim; limss@hallym.ac.kr
}

Received 9 January 2017; Accepted 24 January 2017; Published 23 February 2017

Academic Editor: Kota V. Ramana

Copyright ( 2017 Seung Hwan Hwang et al. This is an open access article distributed under the Creative Commons Attribution License, which permits unrestricted use, distribution, and reproduction in any medium, provided the original work is properly cited.

\begin{abstract}
Stauntonia hexaphylla (Thunb.) Decne. (Lardizabalaceae) leaves (SHL) have been used traditionally as analgesics, sedatives, diuretics, and so on, in China. To date, no data have been reported on the inhibitory effect of SHL and its constituents on rat lens aldose reductase (RLAR) and advanced glycation end products (AGEs). Therefore, the inhibitory effect of compounds isolated from SHL extract on RLAR and AGEs was investigated to evaluate potential treatments of diabetic complications. The ethyl acetate (EtOAC) fraction of SHL extract showed strong inhibitory activity on RLAR and AGEs; therefore, EtOAc fraction (3.0 g) was subjected to Sephadex LH-20 column chromatography, for further fractionation, with $100 \% \mathrm{MeOH}$ solvent system to investigate its effect on RLAR and AGEs. Phytochemical investigation of SHL led to the isolation of seven compounds. Among the isolated compounds, chlorogenic acid, calceolarioside $\mathrm{B}$, luteolin-3'-O- $\beta$-D-glucopyranoside, quercetin-3-O- $\beta$-D-glucopyranoside, and luteolin-7-O- $\beta$-D-glucopyranoside exhibited significant inhibitory activity against RLAR with $\mathrm{IC}_{50}$ in the range of 7.34-23.99 $\mu \mathrm{M}$. In addition, 3-(3,4-dihydroxyphenyl) propionic acid, neochlorogenic acid, and luteolin-3'-O- $\beta$-D-glucopyranoside exhibited the most potent inhibitory activity against formation of AGEs, with an $\mathrm{IC}_{50}$ value of $115.07-184.06 \mu \mathrm{M}$, compared to the positive control aminoguanidine $(820.44 \mu \mathrm{M})$. Based on these findings, SHL dietary supplements could be considered for the prevention and/or treatment of diabetes complication.
\end{abstract}

\section{Introduction}

Diabetic complications including neuropathy, nephropathy, cataracts, and retinopathy are considered to result from the accumulation of sorbitol, which is obtained from reduction of glucose by the catalytic activity of aldose reductase (AR, EC 1.1.1.21) in the polyol pathway. Sorbitol is subsequently metabolized to fructose by sorbitol dehydrogenase [1]. The increased polyol pathway flux leads to the accumulation of sorbitol in the lens fiber, causing an influx of water, generation of osmotic stress, and formation of cataracts which is the leading cause of blindness worldwide in patients with diabetes. Therefore, AR inhibition represents a key point for the prevention and attenuation of long-term diabetic complications [2].

Advanced glycation end products (AGEs) are proteins or lipids that become glycated after exposure to sugars. AGEs are nonenzymatic adducts of protein, lipids, and nucleic acids, formed in a time-dependent manner in a prooxidant environment, especially when the target molecules are slowly metabolized and the levels of aldoses are high [3]. In particular, during hyperglycemia, body proteins undergo increased glycation, where glucose reacts nonenzymatically with protein amino groups to form a labile Schiff base that rearranges to a stable Amadori product. The formation and accumulation of AGEs in many different cell types affect 
TABLE 1: Inhibitory effect of crude extract and fractions of Stauntonia hexaphylla leaves on rat lens aldose reductase (RLAR), advanced glycation end products (AGEs), and antioxidant (DPPH).

\begin{tabular}{|c|c|c|c|c|}
\hline \multirow{2}{*}{\multicolumn{2}{|c|}{ Extract and fractions }} & \multicolumn{3}{|c|}{$\mathrm{IC}_{50}(\mu \mathrm{g} / \mathrm{mL})^{\mathrm{a}}$} \\
\hline & & RLAR & AGEs & DPPH \\
\hline Extract & $70 \% \mathrm{EtOH}$ & $34.52 \pm 2.21$ & $422.51 \pm 36.77$ & $193.19 \pm 19.01$ \\
\hline \multirow{5}{*}{ Fractions } & Hex & $31.24 \pm 2.24$ & $270.51 \pm 26.77$ & $>2000$ \\
\hline & $\mathrm{CH}_{2} \mathrm{Cl}_{2}$ & $24.87+0.98$ & $222.53 \pm 11.25$ & $420.28 \pm 41.23$ \\
\hline & EtOAc & $6.90 \pm 0.34$ & $50.07 \pm 4.21$ & $63.00 \pm 6.20$ \\
\hline & $n-\mathrm{BuOH}$ & $14.98 \pm 1.01$ & $100.90 \pm 9.32$ & $254.84 \pm 21.52$ \\
\hline & Water & $>50$ & $>500$ & $1109.59 \pm 98.17$ \\
\hline \multirow{3}{*}{ Positive controls } & Quercetin $^{\mathrm{b}}$ & $5.48 \pm 0.44$ & - & - \\
\hline & Aminoguanidine $^{c}$ & - & $90.70 \pm 8.71$ & - \\
\hline & L-ascorbic acid ${ }^{\mathrm{d}}$ & - & - & $9.58 \pm 7.89$ \\
\hline
\end{tabular}

${ }^{\mathrm{a}} \mathrm{The} \mathrm{IC}_{50}$ value was defined as a mean \pm SEM of half-maximal inhibitory concentration from three independent experiments performed in duplicate.

${ }^{\mathrm{b}}$ Quercetin is the positive control for aldose reductase.

${ }^{c}$ Aminoguanidine is the positive control for advanced glycation end products.

${ }^{d} \mathrm{~L}$-ascorbic acid is the positive control for DPPH.

extracellular and intracellular structure and functions by inducing oxidative stress [4].

Stauntonia hexaphylla (Thunb.) Decne. (Lardizabalaceae) is widely distributed as thickets in lowlands and foothills of warmer regions of Korea, Japan, and China. S. hexaphylla has been traditionally used in China as analgesic, sedative, and diuretic, among other purposes [5]. In recent years, much attention has been paid to $S$. hexaphylla mainly due to its various biological activities, particularly, anti-HIV-1 [6], antiinflammatory [7], and cytotoxic properties [8]. The known chemical constituents of $S$. hexaphylla include triterpenoids, glucosides, flavonoids, phenylpropanoids, phenolic glucosides, and chlorogenic acid analogues [9].

To date, however, no data are available on the inhibitory effects of the S. hexaphylla leaves' (SHL) constituents on rat lens aldose reductase (RLAR) and AGEs. In the present study, we investigated the inhibitory effect of compounds isolated from SHL on RLAR and AGEs to evaluate their potential to treat diabetic complications.

\section{Materials and Methods}

2.1. Chemicals and Reagents. DL-Glyceraldehyde, reduced form of nicotinamide adenine dinucleotide phosphate (NADPH), bovine serum albumin, methylglyoxal, 2,2diphenyl-1-picrylhydrazyl (DPPH), aminoguanidine, Lascorbic acid, and quercetin used in this study were purchased from Sigma (St. Louis, MO, USA). All other chemicals and reagents used were of analytical grade.

2.2. Instruments. ${ }^{1} \mathrm{H}$ and ${ }^{13} \mathrm{C}$ NMR spectra and correlation NMR spectra such as COSY, HMBC, and HMQC were obtained from an Avance DPX 400 (or 600) spectrometer (Bruker, Madison, WI, USA). These were obtained at operating frequencies of $400 \mathrm{MHz}$ (or 600) $\left({ }^{1} \mathrm{H}\right.$ ) and 100 (or 150) $\mathrm{MHz}\left({ }^{13} \mathrm{C}\right)$ with $\mathrm{CD}_{3} \mathrm{OD},\left(\mathrm{CD}_{3}\right)_{2} \mathrm{SO}$, and TMS were used as internal standards; chemical shifts were reported in $\delta$ values. The molecular mass was measured using the Voyager
DE STR matrix assisted laser desorption/ionization time-offlight (MALDI-TOF) mass spectrometer (MS, Applied Biosystems, Foster City, CA, USA), the low resolution electronic impact (EI) MS equipped JMS-700 (Tokyo, Japan). The compounds were dissolved in methanol $(\mathrm{MeOH})$ and mixed with a matrix, $\alpha$-cyano- 4 -hydroxycinnamic acid. The ratio of the amount of the sample and matrix was $1: 1(\mathrm{v} / \mathrm{v})$. The mixture was spotted on a stainless steel plate and dried at room temperature. After the water vaporized, MALDITOF analysis was performed with an accelerating voltage of $20 \mathrm{kV}$. The low resolution MS was operated in the negativeion mode with ion source at $250^{\circ} \mathrm{C}$ and $\mathrm{EI}$ at $70 \mathrm{eV}$ with direct insertion probe and the mass range in $50-600 \mathrm{~m} / \mathrm{z}$. Fast atom bombardment (FAB) MS was recorded in the negative form using $m$-nitrobenzyl alcohol as matrix in a JEOL JMSAX 505-WA spectrometer (Tokyo, Japan). Column chromatography procedures were performed on Sephadex LH-20 (GE Healthcare, Uppsala, Sweden).

2.3. Plant Material and Preparation of Extract. Dried SHL were purchased from Dae-Kwang Herb Medicine Co., Ltd. (Chuncheon, Korea) and the voucher specimen (number 2016-RIC-0321) was deposited at the Regional Innovation Center, Hallym University, Korea. The specimen was authenticated by Emeritus Prof. H. J. Chi, Seoul National University, Korea.

2.4. Extraction, Fractionation, and Isolation. The dried SHL $(1 \mathrm{~kg})$ was extracted with $70 \%$ ethanol $(2 \mathrm{~L} \times 2$ times $)$ for $2 \mathrm{~h}$ at room temperature. The combined filtrates were concentrated to dryness in vacuo at $40^{\circ} \mathrm{C}$. The dried extract was suspended $(160 \mathrm{~g})$ in distilled water and partitioned sequentially with $n$-hexane (Hex, $3.8 \mathrm{~g}$ ), methylene chloride $\left(\mathrm{CH}_{2} \mathrm{Cl}_{2}, 10.02 \mathrm{~g}\right)$, ethyl acetate (EtOAc, $\left.13.54 \mathrm{~g}\right), n$-butanol $(n-\mathrm{BuOH}, 62.29 \mathrm{~g})$, and water residue (water, $60.69 \mathrm{~g})$. The EtOAc fraction showed strong inhibitory effect on RLAR and AGEs (Table 1); therefore, EtOAc fraction ( $3 \mathrm{~g}$ ) was chromatographed over a Sephadex LH-20 column using 
<smiles>O=C(O)CCc1ccc(O)c(O)c1</smiles>

3-(3,4-Dihydroxyphenyl)-propionic acid (1)<smiles>O=C(/C=C/c1ccc(O)c(O)c1)OCC(O)C(O)OCCOC(O)C(O)C(O)CO</smiles>

Calceolarioside B (4)<smiles>O=C(O)CC1(O)CCC(O)C(O)C1O</smiles>

Quinic acid<smiles>CC(=O)/C=C/c1ccc(O)c(O)c1</smiles>

Caffeoyl
Chlorogenic acid (2): $\mathrm{R}_{3}=$ caffeoyl; $\mathrm{R}_{5}=\mathrm{H}$

Neochlorogenic acid (3): $\mathrm{R}_{3}=\mathrm{H} ; \mathrm{R}_{5}=$ caffeoyl<smiles></smiles>

Luteolin-3'-O- $\beta$-D-glucopyranoside (5): $\mathrm{R}_{1}=\mathrm{OH} ; \mathrm{R}_{2}=\mathrm{H} ; \mathrm{R}_{3}=\mathrm{O}-\beta$-D-glucopyranose Quercetin-3-O- $\beta$-D-glucopyranoside (6): $\mathrm{R}_{1}=\mathrm{OH} ; \mathrm{R}_{2}=\mathrm{O}-\beta$-D-glucopyranose; $\mathrm{R}_{3}=\mathrm{OH}$ Luteolin-7-O- $\beta$-D-glucopyranoside (7): $\mathrm{R}_{1}=\mathrm{O}-\beta$-D-glucopyranose; $\mathrm{R}_{2}=\mathrm{H} ; \mathrm{R}_{3}=\mathrm{OH}$

FIGURE 1: Chemical structures of the compounds isolated from Stauntonia hexaphylla leaves.

$\mathrm{MeOH}$ as the eluent to obtain eight pooled fractions (SHLFs $1-8)$. SHLFs 1 and 2 were further fractionated using Sephadex LH-20 column chromatography and $\mathrm{MeOH}-\mathrm{H}_{2} \mathrm{O}(4: 1, \mathrm{v} / \mathrm{v})$ as the eluent to give compounds $\mathbf{1}(4.8 \mathrm{mg}), \mathbf{2}(23.9 \mathrm{mg}), \mathbf{3}$ (18.6 mg), 4 (9.4 mg), and $\mathbf{5}(10.3 \mathrm{mg})$. SHLF 6 was subjected to Sephadex LH-20 column chromatography with $\mathrm{MeOH}-$ $\mathrm{H}_{2} \mathrm{O}(1: 1, \mathrm{v} / \mathrm{v})$ as the eluent to give compounds $6(6.9 \mathrm{mg})$ and 7 ( $8.5 \mathrm{mg})$ (Figure 1).

2.5. Preparation of Aldose Reductase. Crude RLAR was prepared as follows: lenses weighing $250-280 \mathrm{~g}$ were removed from Sprague-Dawley rats and frozen at $-70^{\circ} \mathrm{C}$ until use. Noncataractous transparent lenses were pooled and a homogenate was prepared in $0.1 \mathrm{M}$ phosphate buffer saline ( $\mathrm{pH}$ 6.2). RLAR homogenate was then centrifuged at $9600 \times \mathrm{g}$ for $20 \mathrm{~min}$. After centrifugation in a refrigerated centrifuge, the supernatant was collected and used as the RLAR. All procedures were carried out at $4^{\circ} \mathrm{C}[10]$.

2.6. In Vitro Determination of RLAR Inhibition. RLAR activity was assayed spectrophotometrically by measuring the decrease in the absorption of NADPH at $340 \mathrm{~nm}$ over a 4 min period using DL-glyceraldehyde as the substrate. Each $1.0 \mathrm{~mL}$ cuvette contained equal units of the enzyme, $0.1 \mathrm{M}$ sodium phosphate buffer ( $\mathrm{pH}$ 6.2), 0.3 mM NADPH, with or without $10 \mathrm{mM}$ of the substrate, and an inhibitor [11]. The concentration of inhibitors causing 50\% inhibition of enzyme activity $\left(\mathrm{IC}_{50}\right)$ was calculated from the least squares regression line of the logarithmic concentrations plotted against the residual activity.

2.7. Bovine Serum Albumin-Methylglyoxal Assay on AGEs Formation. Bovine serum albumin $(50 \mathrm{mg} / \mathrm{mL})$ was incubated with methylglyoxal $(100 \mathrm{mM})$ in sodium phosphate buffer $(0.1 \mathrm{M}, \mathrm{pH} 7.4)$ in the presence of various concentrations of the compounds (including a control) at $37^{\circ} \mathrm{C}$ for $24 \mathrm{~h}$. Then the fluorescent intensity was measured at an excitation wavelength of $355 \mathrm{~nm}$ and an emission wavelength of $460 \mathrm{~nm}$ with a luminescence spectrometer LS50B (Perkin-Elmer Ltd., Buckinghamshire, England) [4]. The dimethylsulfoxide used as vehicle was found to have no effect on the reaction. All reagents and samples were sterilized by filtration through $0.2 \mathrm{~mm}$ membrane filters.

2.8. DPPH Assay. The stable free radical, DPPH, was used to determine the free radical scavenging activity of the SHL extracts. Briefly, a $0.32 \mathrm{mM}$ solution of DPPH in methanol was prepared, and then $180 \mu \mathrm{l}$ of this solution was mixed with $30 \mu \mathrm{l}$ of each sample $(1.0$ to $5.0 \mathrm{mg} / \mathrm{mL}$ in methanol). After $15 \mathrm{~min}$ of incubation in the dark, the decrease in the absorbance of the solution was measured at $570 \mathrm{~nm}$ on a microplate reader (EL800 Universal Microplate reader, Bio-Tek instruments, Winooski, VT, USA).

2.9. HPLC Analysis of EtOAc Fraction of SHL. The HPLC analysis was performed on an Agilent 1100 series system equipped with a diode-array detector (DAD, Agilent, Sunnyvale, CA, USA) consisting of a vacuum degasser (G1322A), a quaternary pump (G1311A), an autosampler (G1313A), a thermostated column compartment (G1316A), and a DAD (G1315B) system. Separation was achieved on an Eclipse XDB-phenyl column $(150 \mathrm{~mm} \times 4.6 \mathrm{~mm}, 3.5 \mu \mathrm{m})$, coupled with a guard column, at $30^{\circ} \mathrm{C} .10 \mu \mathrm{l}$ of samples was injected into the system. The samples were eluted with acidified water ( $0.1 \%$ trifluoroacetic acid, A) and methanol (B), at 
a flow rate of $0.7 \mathrm{~mL} / \mathrm{min}$. The optimized gradient chromatographic conditions were as follows: $5-100 \% \mathrm{~B}$ at $0-$ $32 \mathrm{~min} ; 100-5 \% \mathrm{~B}$ at $32-35 \mathrm{~min}$; isocratic $5 \% \mathrm{~B}$ at $35-40 \mathrm{~min}$. The detector monitored the eluent at wavelength $254 \mathrm{~nm}$. Calibration curves were peak area versus concentration for each standard solution. Serially diluted solutions of the seven standard compounds prepared in the range of $1,10,25$, 50,75 , and $100 \mu \mathrm{g} / \mathrm{mL}$ were injected into the HPLC for quantification. The limit of quantification (LOQ) was determined as the concentration with a signal-to-noise ratio of ten.

\section{Results}

3.1. Structural Determination of Isolated Compounds. Seven compounds were separated from EtOAc fraction by Sephadex LH-20 column chromatography. These compounds were identified by comparing ${ }^{1} \mathrm{H}$ and ${ }^{13} \mathrm{C}$ NMR spectra and correlation NMR spectra such as COSY, HMBC, and HMQC with previously reported data and EI-, FAB-, and MALDITOF MS. The seven compounds are compounds 1 (3-(3,4dihydroxyphenyl)-propionic acid) [12], 2 (chlorogenic acid) [10], 3 (neochlorogenic acid) [13], 4 (calceolarioside B) [14], 5 (luteolin-3'-O- $\beta$-D-glucopyranoside) [15], 6 (quercetin3 - $O$ - $\beta$-D-glucopyranoside) [16], and 7 (luteolin-7- $O-\beta$-Dglucopyranoside) [17].

Compound 1. EI-MS $m / z 183[\mathrm{M}+\mathrm{H}]^{+}, 165\left[^{\mathrm{M}-\mathrm{OH}}\right]^{+}, 138$ $[\mathrm{M}-\mathrm{COOH}]^{+}$. UV (MeCN, $\left.\lambda_{\max } \mathrm{nm}\right) 229,254,290 .{ }^{1} \mathrm{H}$ NMR $\left(400 \mathrm{MHz}, \mathrm{CD}_{3} \mathrm{OD}\right): \delta 6.87(1 \mathrm{H}, \mathrm{d}, J=1.84 \mathrm{~Hz}, \mathrm{H}-2), 6.69(1 \mathrm{H}$, $\mathrm{d}, J=8.01 \mathrm{~Hz}, \mathrm{H}-5), 6.64(1 \mathrm{H}, \mathrm{dd}, J=8.01$ and $1.84 \mathrm{~Hz}, \mathrm{H}-6)$, $2.77(2 \mathrm{H}, \mathrm{t}, J=7.38 \mathrm{~Hz}, \mathrm{H}-7 \mathrm{ab}), 2.52(2 \mathrm{H}, \mathrm{t}, J=7.70 \mathrm{~Hz}, \mathrm{H}-$ 8b). ${ }^{13} \mathrm{C} \mathrm{NMR}\left(100 \mathrm{MHz}, \mathrm{CD}_{3} \mathrm{OD}\right): \delta 174.40$ (C-9), 146.08 (C3), 144.61 (C-4), 131.40 (C-1), 121.26 (C-6), 117.07 (C-2), 116.36 (C-5), 36.71 (C-8), 29.94 (C-7).

Compound 2. MALDI-TOF MS $m / z 377.1275[\mathrm{M}+\mathrm{Na}]^{+}$, $400.1173[\mathrm{M}+2 \mathrm{Na}]^{+}$. UV $\left(\mathrm{MeCN}, \lambda_{\max } \mathrm{nm}\right) 298,346 .{ }^{1} \mathrm{H}$ NMR (400 MHz, CD 3 OD): $\delta 7.55(1 \mathrm{H}, \mathrm{d}, J=15.91 \mathrm{~Hz}, \mathrm{H}-$ $\left.7^{\prime}\right), 7.04\left(1 \mathrm{H}, \mathrm{d}, J=1.80 \mathrm{~Hz}, \mathrm{H}-2^{\prime}\right), 6.94(1 \mathrm{H}, \mathrm{dd}, J=8.21 \mathrm{~Hz}$ and $\left.J=1.80 \mathrm{~Hz}, \mathrm{H}-6^{\prime}\right), 6.77\left(1 \mathrm{H}, \mathrm{d}, J=8.23 \mathrm{~Hz}, \mathrm{H}-5^{\prime}\right), 6.26$ $\left(1 \mathrm{H}, \mathrm{d}, J=15.92 \mathrm{~Hz}, \mathrm{H}-8^{\prime}\right), 5.34(1 \mathrm{H}, \mathrm{m}, \mathrm{H}-3), 4.17(1 \mathrm{H}, \mathrm{m}, \mathrm{H}-$ 5), $3.72(1 \mathrm{H}, \mathrm{dd}, J=8.51 \mathrm{~Hz}$ and $J=3.03 \mathrm{~Hz}, \mathrm{H}-4), 2.21(2 \mathrm{H}$, m, H-6), 2.05 (2H, m, H-2). $\left.{ }^{13} \mathrm{C} \mathrm{NMR} \mathrm{(100} \mathrm{MHz,} \mathrm{CD}_{3} \mathrm{OD}\right): \delta$ 175.95 (C-7), $167.34\left(\mathrm{C}-9^{\prime}\right), 148.15\left(\mathrm{C}-4^{\prime}\right), 145.68\left(\mathrm{C}-7^{\prime}\right), 145.39$ $\left(\mathrm{C}-3^{\prime}\right), 126.41\left(\mathrm{C}-1^{\prime}\right), 121.59\left(\mathrm{C}-6^{\prime}\right), 115.10\left(\mathrm{C}-8^{\prime}\right), 113.90\left(\mathrm{C}-5^{\prime}\right)$, $113.82\left(\mathrm{C}-2^{\prime}\right), 74.92$ (C-1), 72.24 (C-3), 70.63 (C-4), 70.09 (C5), 37.59 (C-6), 36.87 (C-2).

Compound 3. MALDI-TOF MS $m / z 377.0947[\mathrm{M}+\mathrm{Na}]^{+}$, $400.0845[\mathrm{M}+2 \mathrm{Na}]^{+}$. UV $\left(\mathrm{MeCN}, \lambda_{\max } \mathrm{nm}\right) 243,328 .{ }^{1} \mathrm{H}$ $\operatorname{NMR}\left(600 \mathrm{MHz}, \mathrm{CD}_{3} \mathrm{OD}\right): \delta 7.58\left(1 \mathrm{H}, \mathrm{d}, J=15.93 \mathrm{~Hz}, \mathrm{H}-7^{\prime}\right)$, $7.04\left(1 \mathrm{H}\right.$, br s, $\left.\mathrm{H}-2^{\prime}\right), 6.93\left(1 \mathrm{H}, \mathrm{d}, J=8.01 \mathrm{~Hz}, \mathrm{H}-6^{\prime}\right), 6.77(1 \mathrm{H}$, $\left.\mathrm{d}, J=8.03 \mathrm{~Hz}, \mathrm{H}-5^{\prime}\right), 6.31\left(1 \mathrm{H}, \mathrm{d}, J=15.94 \mathrm{~Hz}, \mathrm{H}-8^{\prime}\right), 5.36$ (1H, br s, H-5), 4.13 (1H, s, H-3), $3.66(1 \mathrm{H}, \mathrm{m}, \mathrm{H}-4), 2.14(2 \mathrm{H}$, m, H-6), 1.97 (2H, m, H-2). $\left.{ }^{13} \mathrm{C} \mathrm{NMR} \mathrm{(125} \mathrm{MHz,} \mathrm{CD}_{3} \mathrm{OD}\right): \delta$

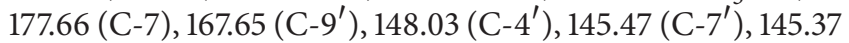
$\left(\mathrm{C}-3^{\prime}\right), 126.59\left(\mathrm{C}-1^{\prime}\right), 121.52\left(\mathrm{C}-6^{\prime}\right), 115.10\left(\mathrm{C}-2^{\prime}\right), 114.42\left(\mathrm{C}-5^{\prime}\right)$,
$113.78\left({\mathrm{C}-8^{\prime}}^{\prime}\right), 78.93(\mathrm{C}-1), 73.18$ (C-5), 71.56 (C-4), 67.20 (C-3), 39.78 (C-6), 35.43 (C-2).

Compound 4. MALDI-TOF MS $m / z 501.1398[\mathrm{M}+\mathrm{Na}]^{+}$, $524.1296[\mathrm{M}+2 \mathrm{Na}]^{+}$. UV $\left(\mathrm{MeCN}, \lambda_{\max } \mathrm{nm}\right) 218,327 .{ }^{1} \mathrm{H}$ NMR $\left(400 \mathrm{MHz}, \mathrm{CD}_{3} \mathrm{OD}\right): \delta 7.55(1 \mathrm{H}, \mathrm{d}, J=15.86 \mathrm{~Hz}, \mathrm{H}-$ $\left.7^{\prime \prime}\right), 7.03\left(1 \mathrm{H}, \mathrm{d}, J=1.92 \mathrm{~Hz}, \mathrm{H}-2^{\prime \prime}\right), 6.88(1 \mathrm{H}, \mathrm{dd}, J=8.24$ and $\left.1.92 \mathrm{~Hz}, \mathrm{H}-6^{\prime \prime}\right), 6.76\left(1 \mathrm{H}, \mathrm{d}, J=8.23 \mathrm{~Hz}, \mathrm{H}-5^{\prime \prime}\right), 6.67(1 \mathrm{H}$, d, $J=1.91 \mathrm{~Hz}, \mathrm{H}-2), 6.63(1 \mathrm{H}, \mathrm{d}, J=8.12 \mathrm{~Hz}, \mathrm{H}-5), 6.53(1 \mathrm{H}$, $\mathrm{dd}, J=8.12$ and $1.92 \mathrm{~Hz}, \mathrm{H}-6), 6.28(1 \mathrm{H}, \mathrm{d}, J=15.87 \mathrm{~Hz}$, $\left.\mathrm{H}-8^{\prime \prime}\right), 4.49\left(1 \mathrm{H}, \mathrm{dd}, J=11.91\right.$ and $\left.1.90 \mathrm{~Hz}, \mathrm{H}-6^{\prime} \mathrm{a}\right), 4.35(1 \mathrm{H}$, br d, $\left.J=5.73 \mathrm{~Hz}, \mathrm{H}-6^{\prime} \mathrm{b}\right), 4.32\left(1 \mathrm{H}, \mathrm{d}, J=8.12 \mathrm{~Hz}, \mathrm{H}-1^{\prime}\right)$, 4.00 (1H, m, H-8a), 3.72 (1H, m, H-8b), 3.55-3.32 (4H, m, H$2^{\prime}, 3^{\prime}, 4^{\prime}$ and $\left.5^{\prime}\right), 2.77(2 \mathrm{H}, \mathrm{m}, \mathrm{H}-7 \mathrm{ab}) .{ }^{13} \mathrm{C} \mathrm{NMR}(100 \mathrm{MHz}$, $\left.\mathrm{CD}_{3} \mathrm{OD}\right): \delta 169.13\left(\mathrm{C}-9^{\prime \prime}\right), 149.57\left(\mathrm{C}-4^{\prime \prime}\right), 147.23\left(\mathrm{C}-7^{\prime \prime}\right), 146.73$ $\left(\mathrm{C}-3^{\prime \prime}\right), 146.08$ (C-3), $144.61(\mathrm{C}-4), 131.40(\mathrm{C}-1), 127.68\left(\mathrm{C}-1^{\prime \prime}\right)$, $123.13\left(\mathrm{C}-6^{\prime \prime}\right), 121.26(\mathrm{C}-6), 117.07$ (C-2), $116.54\left(\mathrm{C}-5^{\prime \prime}\right), 116.36$ $(\mathrm{C}-5), 115.10\left(\mathrm{C}-2^{\prime \prime}\right) 114.83\left(\mathrm{C}-8^{\prime \prime}\right), 104.35\left(\mathrm{C}-1^{\prime}\right), 75.65\left(\mathrm{C}-2^{\prime}\right)$, $75.38\left(\mathrm{C}-5^{\prime}\right), 72.96\left(\mathrm{C}-3^{\prime}\right), 72,32(\mathrm{C}-8), 70.37\left(\mathrm{C}-4^{\prime}\right), 64.61(\mathrm{C}-$ $\left.6^{\prime}\right), 36.65(\mathrm{C}-7)$.

Compound 5. FAB-MS $m / z 449[\mathrm{M}+\mathrm{H}]^{+}, 287[\mathrm{M}+\mathrm{H}-$ glucse $]^{+}$. UV (MeCN, $\left.\lambda_{\max } \mathrm{nm}\right) 265,296,331 .{ }^{1} \mathrm{H} \mathrm{NMR}$ (400 MHz, CD $\mathrm{OD}): \delta 7.42(1 \mathrm{H}, \mathrm{dd}, J=8.17$ and $2.12 \mathrm{~Hz}, \mathrm{H}-$ $\left.6^{\prime}\right), 7.38\left(1 \mathrm{H}, \mathrm{d}, J=2.51 \mathrm{~Hz}, \mathrm{H}-2^{\prime}\right), 6.79(1 \mathrm{H}, \mathrm{d}, J=8.53 \mathrm{~Hz}$, H-5' $\left.{ }^{\prime}\right), 6.62(1 \mathrm{H}, \mathrm{d}, J=1.87 \mathrm{~Hz}, \mathrm{H}-8), 6.58$ (1H, s, H-3), 6.43 $(1 \mathrm{H}, \mathrm{d}, J=1.87 \mathrm{~Hz}, \mathrm{H}-6), 5.32(1 \mathrm{H}, \mathrm{d}, J=7.51 \mathrm{~Hz}, \mathrm{H}-$ $\left.1^{\prime \prime}\right), 4.01-3.53\left(6 \mathrm{H}, \mathrm{m}, \mathrm{H}-2^{\prime \prime}, 3^{\prime \prime}, 4^{\prime \prime}, 5^{\prime \prime}\right.$ and $\left.6^{\prime \prime} \mathrm{ab}\right) .{ }^{13} \mathrm{C} \mathrm{NMR}$ (100 MHz, CD 3 OD): $\delta 187.77$ (C-4), 169.21 (C-7), 166.39 (C-2), 163.58 (C-5), 159.93 (C-9), $156.87\left(\mathrm{C}-4^{\prime}\right), 153.22\left(\mathrm{C}-3^{\prime}\right), 126.74$ $\left(\mathrm{C}-1^{\prime}\right), 121.77\left(\mathrm{C}-6^{\prime}\right), 118.54\left(\mathrm{C}-5^{\prime}\right), 119.87\left(\mathrm{C}-2^{\prime}\right), 107.94(\mathrm{C}-3)$, 108.13 (C-10), 102.15 (C-1" ), 98.22 (C-6), 96.92 (C-8), 76.34 $\left(\mathrm{C}-5^{\prime \prime}\right), 74.80\left(\mathrm{C}-3^{\prime \prime}\right), 73.97\left(\mathrm{C}-2^{\prime \prime}\right), 72.54\left(\mathrm{C}-4^{\prime \prime}\right), 63.33(\mathrm{C}-$ $\left.6^{\prime \prime}\right)$.

Compound 6. FAB-MS $m / z 465[\mathrm{M}+\mathrm{H}]^{+}, 303[\mathrm{M}+$ H-glucse $]^{+}$. UV (MeCN, $\left.\lambda_{\max } \mathrm{nm}\right)$ 257, 359. ${ }^{1} \mathrm{H}-\mathrm{NMR}$ $\left(400 \mathrm{MHz},\left(\mathrm{CD}_{3}\right)_{2} \mathrm{SO}\right): \delta 7.48(1 \mathrm{H}, \mathrm{dd}, J=8.20$ and $1.99 \mathrm{~Hz}$, H- $\left.6^{\prime}\right), 7.47\left(1 \mathrm{H}, \mathrm{d}, J=2.27 \mathrm{~Hz}, \mathrm{H}-2^{\prime}\right), 6.78(1 \mathrm{H}, \mathrm{d}, J=8.19 \mathrm{~Hz}$, $\left.\mathrm{H}-5^{\prime}\right), 6.32(1 \mathrm{H}, \mathrm{d}, J=1.67 \mathrm{~Hz}, \mathrm{H}-8), 6.13(1 \mathrm{H}, \mathrm{d}, J=1.68 \mathrm{~Hz}$, $\mathrm{H}-6), 5.28\left(1 \mathrm{H}, \mathrm{d}, J=7.20 \mathrm{~Hz}, \mathrm{H}-1^{\prime \prime}\right), 3.23-3.41\left(6 \mathrm{H}, \mathrm{m}, \mathrm{H}-2^{\prime \prime}\right.$, $3^{\prime \prime}, 4^{\prime \prime}, 5^{\prime \prime}$ and $\left.6^{\prime \prime} \mathrm{ab}\right) .{ }^{13} \mathrm{C}-\mathrm{NMR}\left(100 \mathrm{MHz}, \delta,\left(\mathrm{CD}_{3}\right)_{2} \mathrm{SO}\right): \delta$ 177.33 (C-4), 164.06 (C-7), 161.18 (C-5), 156.56 (C-9), 156.38 (C-2), $148.37\left(\mathrm{C}-4^{\prime}\right), 144.71\left(\mathrm{C}-3^{\prime}\right), 133.28(\mathrm{C}-3), 121.54\left(\mathrm{C}-1^{\prime}\right)$, $121.14\left(\mathrm{C}-6^{\prime}\right), 116.23\left(\mathrm{C}-5^{\prime}\right), 115.18\left(\mathrm{C}-2^{\prime}\right), 103.92$ (C-10), 101.16 $\left(\mathrm{C}-1^{\prime \prime}\right)$, $98.64(\mathrm{C}-6), 93.54(\mathrm{C}-8), 76.42\left(\mathrm{C}-3^{\prime \prime}\right), 75.87\left(\mathrm{C}-5^{\prime \prime}\right)$, $74.04\left(\mathrm{C}-2^{\prime \prime}\right), 70.53\left(\mathrm{C}-4^{\prime \prime}\right), 66.95\left(\mathrm{C}-6^{\prime \prime}\right)$.

Compound 7. FAB-MS $m / z 449[\mathrm{M}+\mathrm{H}]^{+}, 287[\mathrm{M}+\mathrm{H}-$ glucse $]^{+}$. UV $\left(\mathrm{MeCN}, \lambda_{\max } \mathrm{nm}\right) 256,266,348 .{ }^{1} \mathrm{H} \mathrm{NMR}$ (400 MHz, CD 3 OD): $\delta 7.44(1 \mathrm{H}, \mathrm{dd}, J=8.43$ and $2.02 \mathrm{~Hz}$, H- $\left.6^{\prime}\right), 7.34\left(1 \mathrm{H}, \mathrm{d}, J=1.90 \mathrm{~Hz}, \mathrm{H}-2^{\prime}\right), 6.87(1 \mathrm{H}, \mathrm{d}, J=$ $\left.8.63 \mathrm{~Hz}, \mathrm{H}-5^{\prime}\right), 6.68(1 \mathrm{H}, \mathrm{d}, J=1.82 \mathrm{~Hz}, \mathrm{H}-8), 6.50(1 \mathrm{H}$, s, H-3), $6.45(1 \mathrm{H}, \mathrm{d}, J=1.81 \mathrm{~Hz}, \mathrm{H}-6), 5.29(1 \mathrm{H}, \mathrm{d}, J=$ $\left.7.39 \mathrm{~Hz}, \mathrm{H}-1^{\prime \prime}\right), 4.16-3.42\left(6 \mathrm{H}, \mathrm{m}, \mathrm{H}-2^{\prime \prime}, 3^{\prime \prime}, 4^{\prime \prime}, 5^{\prime \prime}\right.$ and $\left.6^{\prime \prime} \mathrm{ab}\right)$. ${ }^{13} \mathrm{C}$ NMR (100 MHz, CD 3 OD): $\delta 184.38$ (C-4), $166.62(\mathrm{C}-7)$, 165.24 (C-2), 162.41 (C-5), 159.08 (C-9), 151.42 (C-4'), 147.42 $\left(\mathrm{C}-3^{\prime}\right), 123.93\left(\mathrm{C}-1^{\prime}\right), 120.73\left(\mathrm{C}-6^{\prime}\right), 117.19\left(\mathrm{C}-5^{\prime}\right), 114.56\left(\mathrm{C}-2^{\prime}\right)$, 109.54 (C-3), 105.61 (C-10), $104.30\left(\mathrm{C}-1^{\prime \prime}\right), 97.61$ (C-6), 95.40 
TABLE 2: Inhibitory effect of compounds isolated from Stauntonia hexaphylla leaves on rat lens aldose reductase (RLAR).

\begin{tabular}{lc}
\hline Compounds & $\mathrm{IC}_{50}(\mu \mathrm{M})^{\mathrm{a}}$ \\
\hline 3-(3,4-Dihydroxyphenyl)-propionic acid (1) & $>1000$ \\
Chlorogenic acid (2) & $8.35 \pm 0.45$ \\
Neochlorogenic acid (3) & $72.03 \pm 25.77$ \\
Calceolarioside B (4) & $23.99 \pm 2.30$ \\
Luteolin-3'-O- $\beta$-D-glucopyranoside (5) & $7.34 \pm 0.35$ \\
Quercetin-3-O- $\beta$-D-glucopyranoside (6) & $10.40 \pm 1.38$ \\
Luteolin-7-O- $\beta$-D-glucopyranoside (7) $^{\text {Q }}$ & $16.10 \pm 1.20$ \\
\hline Quercetin $^{\mathrm{b}}$ & $18.09 \pm 1.30$ \\
\hline
\end{tabular}

${ }^{\mathrm{a}}$ The $\mathrm{IC}_{50}$ value was defined as a mean \pm SEM of half-maximal inhibitory concentration from three independent experiments performed in duplicate.

${ }^{\mathrm{b}}$ Quercetin is the positive control for aldose reductase.

$(\mathrm{C}-8), 77.53\left(\mathrm{C}-5^{\prime \prime}\right), 75.71\left(\mathrm{C}-3^{\prime \prime}\right), 73.02\left(\mathrm{C}-2^{\prime \prime}\right), 72.20\left(\mathrm{C}-4^{\prime \prime}\right)$, $63.29\left(\mathrm{C}-6^{\prime \prime}\right)$.

3.2. Inhibitory Activity of Isolated Compounds on RLAR. A $70 \%$ ethanol extract of SHL was found to exhibit inhibitory activity against RLAR $(34.52 \mu \mathrm{g} / \mathrm{mL})$, AGEs $(422.51 \mu \mathrm{g} / \mathrm{mL})$, and DPPH $(193.19 \mu \mathrm{g} / \mathrm{mL})$ compared with positive controls quercetin $(5.48 \mu \mathrm{g} / \mathrm{mL})$, aminoguanidine $(90.70 \mu \mathrm{g} / \mathrm{mL})$, and L-ascorbic acid $(9.58 \mu \mathrm{g} / \mathrm{mL})$. The extract was suspended in distilled water and partitioned with with $\mathrm{Hex}, \mathrm{CH}_{2} \mathrm{Cl}_{2}$, EtOAc, n-BuOH, and water residue. Among fractions, the EtOAc fraction exhibited potent inhibitory activity against RLAR with $\mathrm{IC}_{50}$ value of $6.90 \mu \mathrm{g} / \mathrm{mL}$. Therefore, this study focused on the isolation of $\mathrm{AR}$ inhibitors from this fraction (Table 1). The inhibitory activities of the compounds 1-7 isolated from EtOAc fraction were evaluated on RLAR. Of the tested compounds, $\mathbf{2 , 5}, \mathbf{6}$, and 7 showed strong inhibitory activities on RLAR with $\mathrm{IC}_{50}$ value of $8.35,7.34,10.40$, and $16.10 \mu \mathrm{M}$, respectively (Table 2 ), which were higher than that of positive control (quercetin, $18.09 \mu \mathrm{M}$ ). In addition, compounds $\mathbf{3}$ and $\mathbf{4}$ showed inhibitory effect against RLAR with $\mathrm{IC}_{50}$ of 72.03 and $23.99 \mu \mathrm{M}$, respectively.

3.3. Inhibitory Activity of Isolated Compounds on AGEs. The extract and fractions were evaluated for AGEs using a bovine serum albumin-methylglyoxal assay. All the extract and fractions showed significant inhibitory activity with $\mathrm{IC}_{50}$ values ranging from 9.80 to $28.44 \mu \mathrm{g} / \mathrm{mL}$ (Table 1 ). Among the fractions, the EtOAc fraction $(50.07 \mu \mathrm{g} / \mathrm{mL})$ was found to exhibit a similar activity to the positive control, a known AGEs inhibitor (aminoguanidine, $90.70 \mu \mathrm{g} / \mathrm{mL}$ ). Among isolated compounds, AGEs activity results showed that compound $\mathbf{3}$ had the highest inhibitory effect against AGEs formation among isolated compounds with $\mathrm{IC}_{50}$ value of $115.07 \mu \mathrm{M}$; also, compounds $\mathbf{1}$ and $\mathbf{5}$ had $\mathrm{IC}_{50}$ values of 184.06 and $117.80 \mu \mathrm{M}$, respectively (Table 3 ). However, other compounds had no inhibitory effect even at the same concentration when compared to aminoguanidine $(820.44 \mu \mathrm{M})$.

3.4. Antioxidant Activity of Isolated Compounds on ABTS ${ }^{+}$. The antioxidant activity of the fractions and the constituents
TABLE 3: Inhibitory effect of compounds isolated from Stauntonia hexaphylla leaves on advanced glycation end products (AGEs) formation.

\begin{tabular}{lc}
\hline Compounds & $\mathrm{IC}_{50}(\mu \mathrm{M})^{\mathrm{a}}$ \\
\hline 3-(3,4-Dihydroxyphenyl)-propionic acid (1) & $184.06 \pm 17.62$ \\
Chlorogenic acid (2) & $>1000$ \\
Neochlorogenic acid (3) & $115.07 \pm 10.47$ \\
Calceolarioside B (4) & $>1000$ \\
Luteolin-3' $-O$ - $\beta$-D-glucopyranoside (5) & $117.80 \pm 11.46$ \\
Quercetin-3-O- $\beta$-D-glucopyranoside (6) & $>1000$ \\
Luteolin-7-O- $\beta$-D-glucopyranoside (7) & $>1000$ \\
\hline Aminoguanidine ${ }^{\mathrm{b}}$ & $820.44 \pm 79.42$ \\
\hline${ }^{\mathrm{a}}$ The $\mathrm{IC}_{50}$ value was defined as a mean \pm SEM of half-maximal inhibitory \\
concentration from three independent experiments performed in duplicate. \\
${ }^{\mathrm{b}}$ Aminoguanidine is the positive control for advanced glycation end prod- \\
ucts.
\end{tabular}

TABLE 4: Inhibitory effect of compounds isolated from Stauntonia hexaphylla leaves on DPPH radical scavenging activity.

\begin{tabular}{lc}
\hline Compounds & $\mathrm{IC}_{50}(\mu \mathrm{M})^{\mathrm{a}}$ \\
\hline 3-(3,4-Dihydroxyphenyl)-propionic acid (1) & $109.73 \pm 9.39$ \\
Chlorogenic acid (2) & $162.60 \pm 14.39$ \\
Neochlorogenic acid (3) & $>1000$ \\
Calceolarioside B (4) & $94.60 \pm 6.86$ \\
Luteolin-3' $-O$ - $\beta$-D-glucopyranoside (5) & $96.28 \pm 9.19$ \\
Quercetin-3-O- $\beta$-D-glucopyranoside (6) & $122.34 \pm 10.94$ \\
Luteolin-7-O- $\beta$-D-glucopyranoside (7) & $>1000$ \\
\hline L-ascorbic acid & $54.39 \pm 5.73$ \\
\hline${ }^{\mathrm{a}}$ The $\mathrm{IC}_{50}$ value was defined as a mean \pm SEM of half-maximal inhibitory \\
concentration from three independent experiments performed in duplicate. \\
${ }^{\mathrm{b}} \mathrm{L}$-ascorbic acid is the positive control for DPPH radical scavenging activity.
\end{tabular}

was evaluated in vitro by examining the $\mathrm{DPPH}$ radical scavenging activity. As shown in Table 1, the EtOAc fraction of SHL exhibited potent inhibitory activity against $\mathrm{DPPH}\left(\mathrm{IC}_{50}\right.$ value: $63.00 \mu \mathrm{g} / \mathrm{mL}$ ) when compared with that of the positive control (L-ascorbic acid, $9.58 \mu \mathrm{g} / \mathrm{mL}$ ). Among the isolated compounds from the EtOAc fraction, most compounds were found to have inhibitory activities with $\mathrm{IC}_{50}$ in the range of 94.60-162.60 $\mu \mathrm{M}$ (Table 4). Of the tested, compounds 6 and 10 showed strong inhibitory activity with $\mathrm{IC}_{50}$ values of 18.42 and $88.14 \mu \mathrm{M}$, respectively. However, compounds 3 and 7 had no an inhibitory effect even at the same concentration when compared to other compounds.

3.5. Quantification of Isolated Compounds from SHL EtOAc

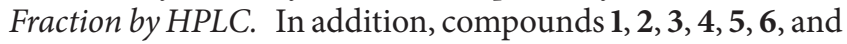
7 in the EtOAc were analyzed with HPLC-DAD (Figure 2). Based on the established method, these compounds (1-7) identified the contents of 3.07, 24.89, 16.80, 4.10, 10.19, 5.14, and $7.77 \%$ in SHL extract, respectively. And, the purity of all compounds isolated was high purities: compound $\mathbf{1}(98.73 \%)$, 

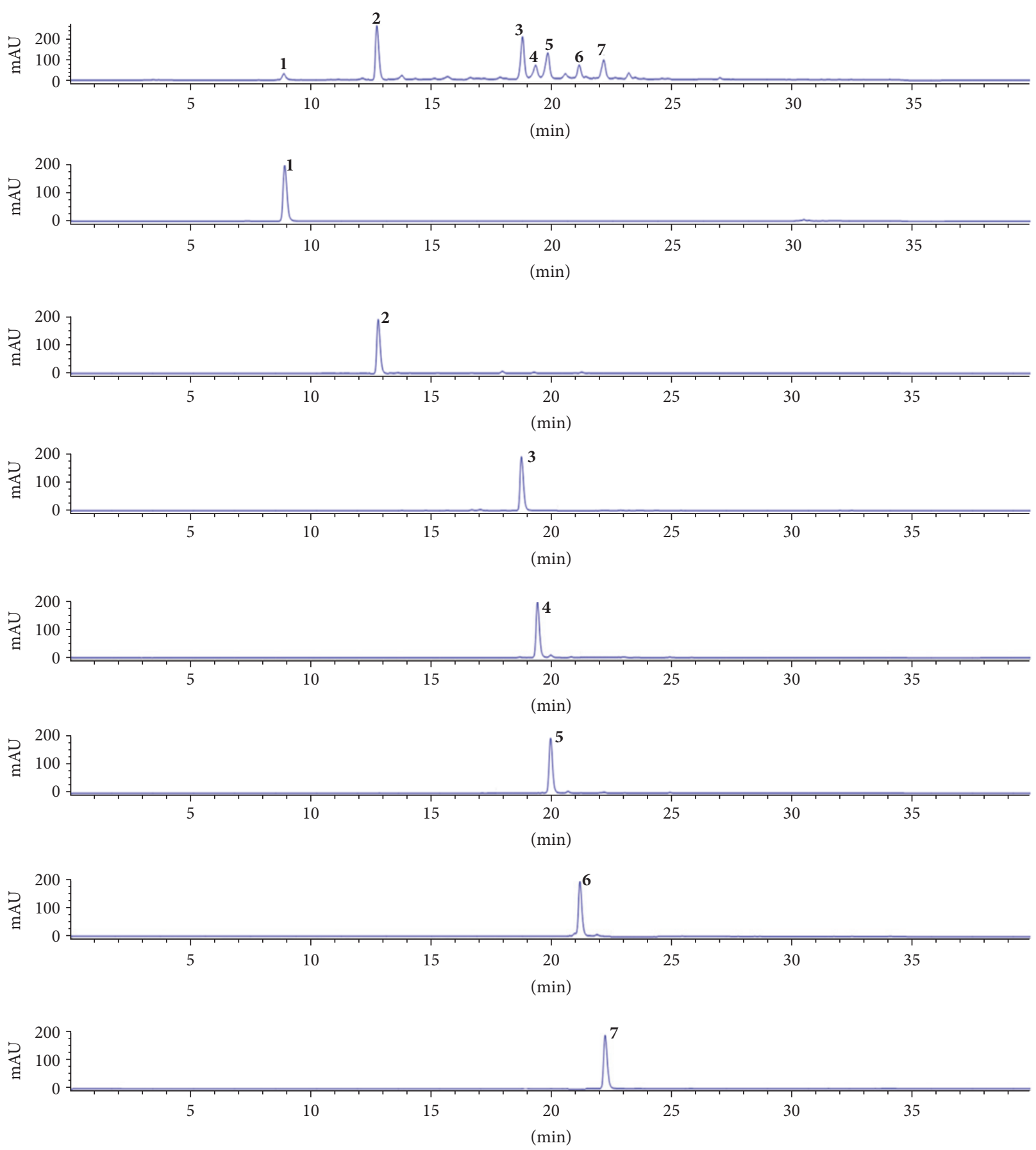

FIGURE 2: HPLC profile of seven compounds isolated from EtOAc fraction of Stauntonia hexaphylla, $70 \%$ ethanol extract at $254 \mathrm{~nm}$.

2 (96.15\%), 3 (98.83\%), 4 (96.83\%), 5 (96.63\%), 6 (95.13\%), and $7(98.36 \%)$, respectively.

\section{Discussion}

The inhibitory behavior of SHL constituents and the related structural activity relationship were investigated using the RLAR assay. RLAR-inhibitory potential of SHL constituents depends on the site of quinic acid and/or other residues of the $-\mathrm{COOH}$ position in the caffeic acid structure (compound $2>4>3$ ). A possible mechanism by which caffeoylquinic acid inhibits RLAR could be related to its structure's action position [11]. In addition, many structural properties of the sugar position in flavonoids that inhibit RLAR have been reported. Increasing the sugar residue at the $3^{\prime}$ position of the $\mathrm{B}$ ring in luteolin (aglycone of compound 5) increases the inhibitory activities against RLAR; in contrast, increasing 
the sugar residue at the $7^{\prime}$ positions of the A ring of luteolin decreases the inhibitory activities against RLAR (compound $5>6>7$ ) [18]. However, AGEs and DPPH results have no significant relationship between structure and their inhibitory activity.

Hyperglycemia plays an important role in the pathogenesis of diabetic complications associated with vascular and nerve damage by several mechanisms such as increased AR-related polyol pathway flux, increased AGEs formation, and excessive oxidation stress [19]. Among them, cataract formation is the leading cause of blindness worldwide in diabetic patients and, especially, must be considered in patients with uncontrolled diabetes. AR inhibitors have received considerable attention because of the proposed involvement of $\mathrm{AR}$ in the pathophysiology of diabetic complications including cataract [20]. In addition, AR-catalyzed formation of sorbitol was also observed in a number of tissues and in diabetes mellitus; increased sorbitol through the polyol pathway does not readily diffuse across cell membranes and the intracellular accumulation of sorbitol has been implicated in the chronic complications of diabetes such as cataract, neuropathy, and retinopathy [21]. These findings suggested that ARIs prevent the conversion of glucose to sorbitol and may have the capacity of preventing and/or treating several diabetic complications [22]. In addition, the formation and accumulation of AGEs in various tissues have been reported to progress at an accelerated rate under hyperglycemic conditions. The formation and accumulation of AGEs will induce oxidative stress and it would have deleterious effects on various cellular functions [23].

Various natural extracts and their constituents have long been used in traditional herbal medicine, particularly in the treatment of diabetes and diabetic complication. In most cases, natural extracts could have less side effects and lower toxicity. Therefore, there is growing interest in using natural products as sources of new drugs [24]. Flavonoid and its derivatives are an interesting chemical group of natural products that are widely distributed in the various plants and most of these compounds are isolated from medicinal plants. A recent study reported that the constituents isolated from Abeliophyllum distichum [25], Zea mays L. [1], and Perilla frutescens [26] showed an inhibitory effect on rat lens AR activity. Polyphenols including rosmarinic acid and caffeic acid ethylene ester isolated from Prunella vulgaris L. displayed therapeutic potential in the prevention and treatment of diabetic complications by inhibiting AR and protein glycation [27]. Our results suggest that SHL and its constituents prevent cataractogenesis by inhibiting AR activity. For this reason, the pursuit for new AR inhibitors of natural origin is highly justified.

\section{Conclusion}

In summary, seven compounds were isolated from the EtOAc fraction of the SHL and the inhibitory effect of the compounds was evaluated against RLAR, AGEs, and DPPH. Consequently, we conclude that SHL extract and its constituents contribute at least in part in RLAR and AGEs inhibition.
Furthermore, our results suggest SHL extract and its constituents as potential natural drugs to treat hyperglycemiainduced cataract and various diabetic complications.

\section{Competing Interests}

The authors have declared that there is no conflict of interests.

\section{Authors' Contributions}

Seung Hwan Hwang and Shin Hwa Kwon have contributed equally to this work.

\section{Acknowledgments}

This research was supported by Basic Science Research Program through the National Research Foundation of Korea (NRF) funded by the Ministry of Education (2015R1D1A1A01059199).

\section{References}

[1] T. H. Kim, J. K. Kim, Y.-H. Kang, J.-Y. Lee, I. J. Kang, and S. S. Lim, "Aldose reductase inhibitory activity of compounds from Zea mays L.," BioMed Research International, vol. 2013, Article ID 727143, 8 pages, 2013.

[2] J. K. Kim, Y. S. Lee, S. H. Kim, Y. S. Bae, and S. S. Lim, "Inhibition of aldose reductase by phenylethanoid glycoside isolated from the seeds of Paulownia coreana," Biological and Pharmaceutical Bulletin, vol. 34, no. 1, pp. 160-163, 2011.

[3] A. Goldin, J. A. Beckman, A. M. Schmidt, and M. A. Creager, "Advanced glycation end products: sparking the development of diabetic vascular injury," Circulation, vol. 114, no. 6, pp. 597605, 2006.

[4] Y. S. Lee, Y.-H. Kang, J.-Y. Jung et al., "Protein glycation inhibitors from the fruiting body of Phellinus linteus," Biological and Pharmaceutical Bulletin, vol. 31, no. 10, pp. 1968-1972, 2008.

[5] H.-B. Wang, R. Mayer, G. Rücker, J.-J. Yang, and D. S. Matteson, "A phenolic glycoside and triterpenoids from Stauntonia hexaphylla," Phytochemistry, vol. 47, no. 3, pp. 467-470, 1998.

[6] Y. Wei, C.-M. Ma, D.-Y. Chen, and M. Hattori, "Anti-HIV1 protease triterpenoids from Stauntonia obovatifoliola Hayata subsp," Phytochemistry, vol. 69, no. 9, pp. 1875-1879, 2008.

[7] H. Gao, F. Zhao, G.-D. Chen et al., "Bidesmoside triterpenoid glycosides from Stauntonia chinensis and relationship to antiinflammation," Phytochemistry, vol. 70, no. 6, pp. 795-806, 2009.

[8] J. Zhao, S.-H. Yim, J.-I. Um et al., "Cytotoxic components in an extract from the leaves and stems of Stauntonia hexaphylla," Natural Product Sciences, vol. 20, no. 2, pp. 130-134, 2014.

[9] X. L. Liu, D. D. Wang, Z. H. Wang, and D. L. Meng, "Diuretic properties and chemical constituent studies on Stauntonia brachyanthera," Evidence-based Complementary and Alternative Medicine, vol. 2015, Article ID 432419, 8 pages, 2015.

[10] H. M. Li, S. H. Hwang, B. G. Kang, J. S. Hong, and S. S. Lim, "Inhibitory effects of Colocasia esculenta (L.) Schott constituents on aldose reductase," Molecules, vol. 19, no. 9, pp. 13212-13224, 2014.

[11] H. N. Yoon, M. Y. Lee, J.-K. Kim, H.-W. Suh, and S. S. Lim, "Aldose reductase inhibitory compounds from Xanthium 
strumarium," Archives of Pharmacal Research, vol. 36, no. 9, pp. 1090-1095, 2013.

[12] A. Mikolasch, E. Hammer, U. Jonas, K. Popowski, A. Stielow, and F. Schauer, "Synthesis of 3-(3,4-dihydroxyphenyl)-propionic acid derivatives by $\mathrm{N}$-coupling of amines using laccase," Tetrahedron, vol. 58, no. 38, pp. 7589-7593, 2002.

[13] N. Nakatani, S.-I. Kayano, H. Kikuzaki, K. Sumino, K. Katagiri, and T. Mitani, "Identification, quantitative determination, and antioxidative activities of chlorogenic acid isomers in prune (Prunus domestica L.)," Journal of Agricultural and Food Chemistry, vol. 48, no. 11, pp. 5512-5516, 2000.

[14] T. Iossifova, B. Vogler, and I. Kostova, "Escuside, a new coumarin-secoiridoid from Fraxinus ornus bark," Fitoterapia, vol. 73, no. 5, pp. 386-389, 2002.

[15] M. A. M. Nawwar, S. A. M. Hussein, and I. Merfort, "Leaf phenolics of Punica granatum," Phytochemistry, vol. 37, no. 4, pp. 1175-1177, 1994.

[16] D. G. An, S. M. Yang, B. G. Kim, and J.-H. Ahn, "Biosynthesis of two quercetin O-diglycosides in Escherichia coli," Journal of Industrial Microbiology \& Biotechnology, vol. 43, no. 6, pp. 841849, 2016.

[17] L.-C. Lin, Y.-F. Pai, and T.-H. Tsai, "Isolation of luteolin and luteolin-7-O-glucoside from Dendranthema morifolium ramat tzvel and their pharmacokinetics in rats," Journal of Agricultural and Food Chemistry, vol. 63, no. 35, pp. 7700-7706, 2015.

[18] S. H. Jung, J. M. Lee, H. J. Lee, C. Y. Kim, E. H. Lee, and B. H. Um, "Aldose reductase and advanced glycation endproducts inhibitory effect of Phyllostachys nigra," Biological and Pharmaceutical Bulletin, vol. 30, no. 8, pp. 1569-1572, 2007.

[19] C. B. Cui, S. J. Jeong, Y. S. Lee, S. O. Lee, I. J. Kang, and S. S. Lim, "Inhibitory activity of caffeoylquinic acid from aerial parts of Artemisia princeps on rat lens aldose reductase and on the formation of advanced glycation end products," Journal of the Korean Society for Applied Biological Chemistry, vol. 52, no. 6, pp. 655-662, 2009.

[20] J. H. Paek and S. S. Lim, "Preparative isolation of aldose reductase inhibitory compounds from Nardostachys chinensis by elution-extrusion counter-current chromatography," Archives of Pharmacal Research, vol. 37, no. 10, pp. 1271-1279, 2014.

[21] Y. S. Lee, S. H. Kim, S. H. Jung, J. K. Kim, P. Cheol-Ho, and S. S. Lim, "Aldose reductase inhibitory compounds from Glycyrrhiza uralensis," Biological and Pharmaceutical Bulletin, vol. 33, no. 5, pp. 917-921, 2010.

[22] X. F. Yin, Y. E. Jeon, H. C. Chung, S. Y. Choung, J.-H. Shim, and I.-J. Kang, "In vitro efficacy evaluation for prevention of diabetes and diabetic complications using Aster sphathulifolius," Food Science and Biotechnology, vol. 24, no. 1, pp. 301-306, 2015.

[23] X. F. Yin, Y. E. Jeon, J.-H. Shim, and I.-J. Kang, "Inhibitory efficacy of Ligularia fischeri against aldose reductase and advanced glycation end products formation," Food Science and Biotechnology, vol. 23, no. 6, pp. 1747-1752, 2014.

[24] S. H. Kim, J. K. Kim, Y. S. Lee, Y. S. Bae, and S. S. Lim, "Inhibitory effect of quercetin-3-O- $\alpha$-L-rhamnopyranoside from Chamaecyparis obtuse on aldose reductase and sorbitol accumulation," Korean Journal of Medicinal Crop Science, vol. 18, no. 5, pp. 305310, 2010.

[25] H. M. Li, J. K. Kim, J. M. Jang, C. B. Cui, and S. S. Lim, "Analysis of the inhibitory activity of Abeliophyllum distichum leaf constituents against aldose reductase by using high-speed counter current chromatography," Archives of Pharmacal Research, vol. 36, no. 9, pp. 1104-1112, 2013.
[26] J. H. Paek, K. H. Shin, Y.-H. Kang, J.-Y. Lee, and S. S. Lim, “Rapid identification of aldose reductase inhibitory compounds from Perilla frutescens," BioMed Research International, vol. 2013, Article ID 679463, 8 pages, 2013.

[27] H. M. Li, J. K. Kim, J. M. Jang, S. O. Kwon, C. B. Cui, and S. S. Lim, "The inhibitory effect of Prunella vulgaris L. on aldose reductase and protein glycation," Journal of Biomedicine and Biotechnology, vol. 2012, Article ID 928159, 7 pages, 2012. 

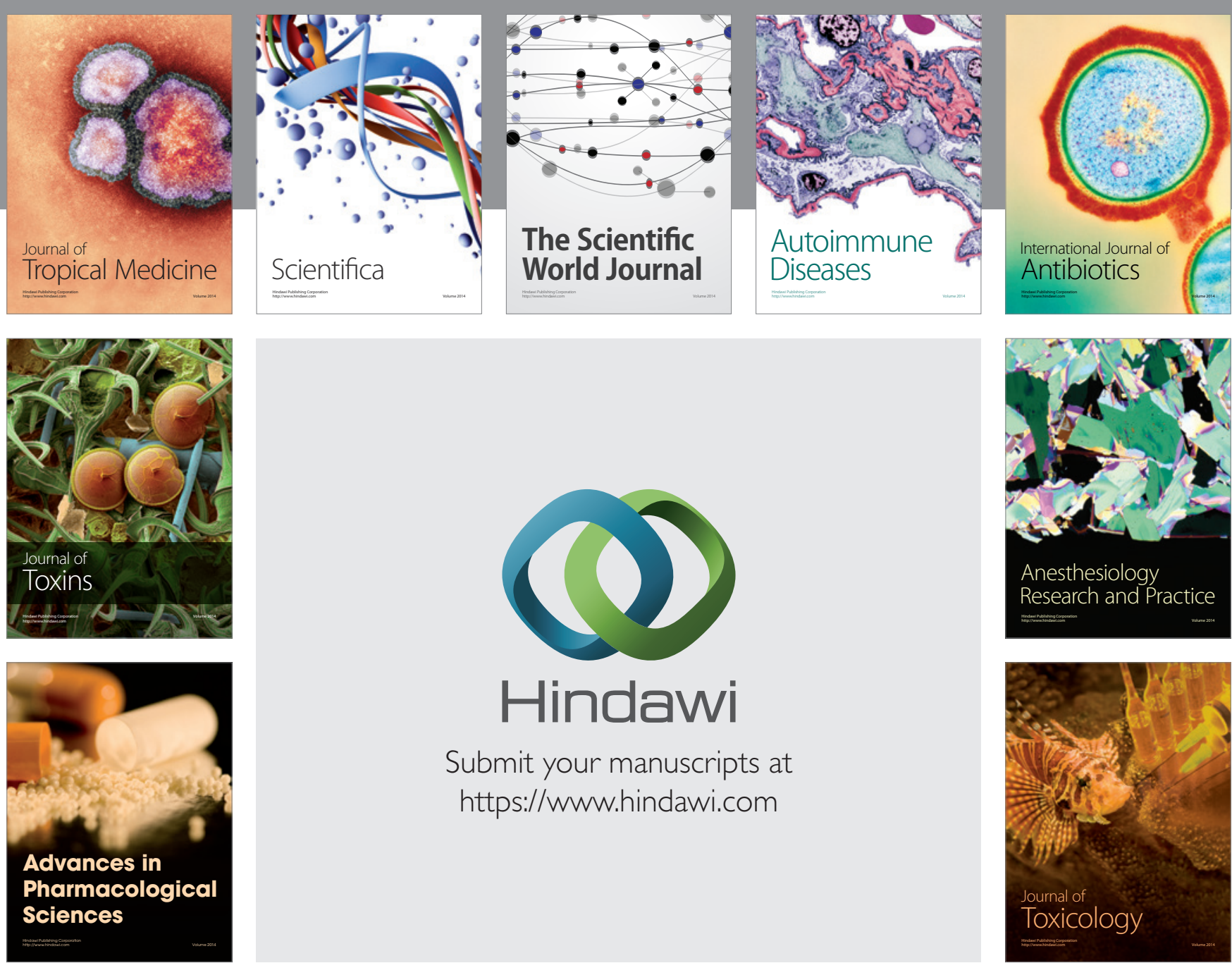

\section{Hindawi}

Submit your manuscripts at

https://www.hindawi.com
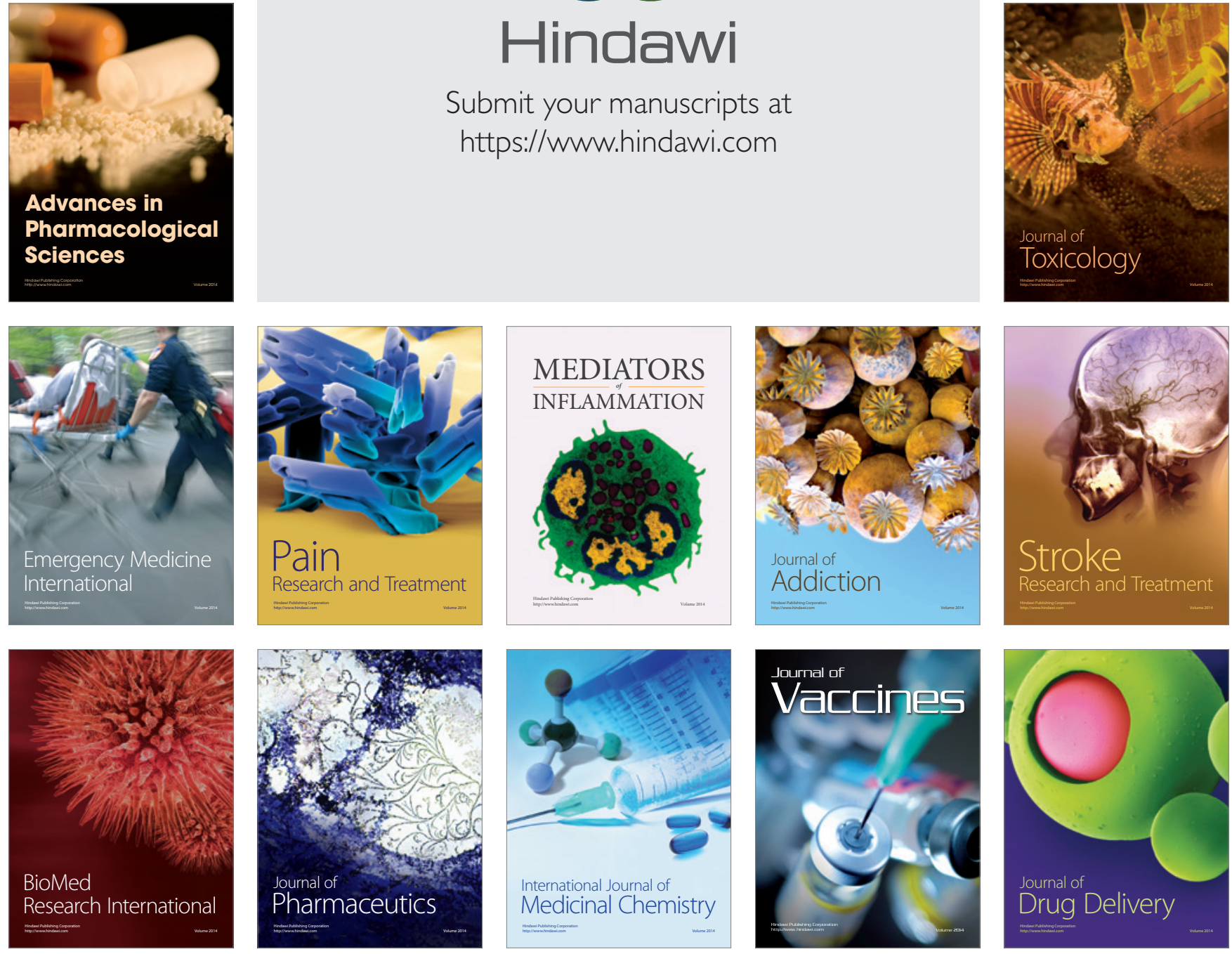